\title{
Nature Makes A Material Difference
}

\section{Christopher Viney}

Materials engineering is eloquently described for the layperson in two classic Penguin paperbacks authored by the pioneering materials technology professor, J.E. Gordon. The two books are The New Science of Strong Materials, or Why You Don't Fall Through the Floor (1968), and the sequel Structures, or Why Things Don't Fall Down (1978). In addition to being valuable as introductory reading, these books are articulate reminders of the origins, mission, and vision of materials engineering at its interdisciplinary best. (It was initially these books, and the apparently unfettered interdisciplinary character of materials science and engineering, that attracted me to the field.) Both books draw attention to the debt that materials engineering owes to physics, mathematics, chemistry, traditional metallurgy, and mechanical engineering. They also remind the reader frequently that useful, practical lessons can and should be drawn from nature. Indeed, Structures starts out with a discussion of nature as a role model for materials design, urging that "for solid quantitative reasons, there is a case for trying to rebuild some part of traditional engineering upon models which may well turn out to be partly biological in inspiration...."

Far-sighted sentiments such as these have not led to timely, appropriate biological coursework in materials curricula, despite the fact that nature has prevailed and made a serious impact on materials anyway! Materials synthesis in particular has benefitted from accepting biological ideas. Bacterial action is exploited directly in heavy-metal extraction, and in recovering heavy metals from industrial effluent. Bacteria can be genetically altered to synthesize new protein polymers that are designed de novo to exhibit pre-determined properties. Monodisperse, submicrometer-size ceramic particles can be precipitated inside lipid membrane-bound vesicles in a process that owes its conceptual origin to the membrane-bound magnetite particles synthesized by magnetotactic bacteria.

Beyond synthesis, there is much to be learned from how nature optimizes micro- structures to achieve particular combinations of properties. Stiff, low-density cellular and honeycomb materials owe their inspiration partly to wood and bone. The microstructural basis of the high strength, stiffness, and toughness achieved by the dragline silk of spiders is a continuing research topic in several laboratories. As symbiosis between the physical and life sciences progresses, structureproperty characterization increasingly reveals that the bulk properties of natural materials depend greatly on their hierarchical microstructure. It is not structure as resolved by any one type of microscopy that is significant, but microstructure at all scales ranging from the arrangement of individual atoms or molecules up to and including the macroscopic shape of the product. This has been amply demonstrated in studies of biological materials that are as diverse as silk, collagen, plant cell walls, rhinoceros horn, and nacre. In the case of collagen, the large differences in the arrangement of molecules-not their relatively minor difference in chemical composition-are responsible for the dissimilar properties of tendons and corneas.

\section{Meaningful biomimicry identifies selected, worthwhile attributes to copy from a natural material.}

The lessons taught by silk go beyond widening the repertoire of known relationships between monomer sequence, hierarchical microstructure, and optimized properties for polymeric materials. A more radical lesson is obtained from studying how silkworms and spiders manage to process aqueous protein solutions into water-insoluble silk fiber. Spinning apparently causes the protein to become insoluble not as a result of chemical changes, but as a result of conformational (i.e., physical) changes that are induced by shear. Such a molecular-level understanding of the natural process will of course facilitate spinning of silklike proteins from biotechnological sources. More generally, however, it is hoped that this knowledge will be used to process other types of high-performance polymer into fibers or films from aqueous solution at room temperature.

Nature also offers many device-oriented lessons. One developing research area concerns the pores that can self-assemble from protein molecules dissolved in a lipid bilayer membrane. The promise of tailorable pore charge and diameter suggests applications for supported membranes in separation systems, electronic devices, and sensors. Looking even further ahead, one can speculate that more efficient pathways for materials synthesis will develop from an understanding of the spatially organized chemistry that is typical of natural biochemical processes. (In nature, thousands of distinct chemical reactions can occur simultaneously in a single, specialized, micron-size cell, achieving what can be thought of as "parallel chemical processing"). Also, an understanding of natural materials synthesis at this level of sophistication may be necessary to produce truly "smart" synthetic materials that have localized functionalities enabling them to sense and respond to changes in their surroundings.

An emerging discipline can generate a plethora of jargon that may be inconsistently or redundantly used by different groups active within that discipline. Thus the materials lexicon is being infused with a rapid succession of "bio-buzzwords" including "bioremediation," "bioduplication," "biomaterial," "biomolecular" material, "biosynthesized" material, "bioderived" material, "bioinspired" material, and "biomimetic" material. Such proliferation of language may be regarded as a sign of healthy activity and rapid progress: there is little time for perceived taxonomic trivia when the broader concepts are new and exciting. All of these words attempt to describe positive aspects of biological knowledge interacting with materials engineering. A distinct danger of overrigorously delineating active research fields is that one could lapse into accepting limits on the range of intellectual input. The growth, or even survival, of a sciencebased field depends on its boundaries being fuzzy. At the same time, concern is justified if new words are being generated merely to attract media attention or grant support. The elastic limit of credibility also is exceeded when such buzzwords are consistently touted in a context that extols only 
the unprecedented virtues of the implicated material!

There are occasions (such as in this editorial) when there is a need for a single word to encompass all the engineering materials that owe a substantial debt traceable to biological knowledge. Such a broad class of materials would currently include those that have benefitted from an idea first learned in studies of materials synthesized by animals, plants, bacteria, or viruses. Also included would be materials that are partially or completely synthesized by a natural or genetically altered organism. I use "bioemanent" as a designation for materials that are impacted significantly by knowledge emanating from the biological sciences. This designation is intended to be useful only to the extent that it economically enables reference to a particular, broad, interesting class of engineering materials; there is no implied judgement of superiority relative to other classes of materials. ker to meet their immediate needs as dictated by equally slow changes in the environment. (It can also be argued, however, that every synthetic material invented by Homo sapiens simply demonstrates the innovative leaps that can be made by a suitably complex and organized collection of biological molecules!)

Nature's limitations have surfaced in exchanges between some champions and some equally passionate detractors of the term "biomimetic." In at least one respect, the term is potentially misleading. It can be (and has been) misunderstood that the proponents of biomimetics would like to copy all aspects of a natural material: ingredients, processing history, microstructure, and properties. There is, of course, little point in copying all these aspects of á material simultaneously-one would then be a victim of all the limitations of that material. For example, researchers involved in seashell biomimetics would be quite misguided to actually copy the detailed

\section{Some early flying machines were constructed to have flapping wings. Their failure constitutes an exercise in misdirected biomimetics. But learning that the curvature of a wing matters, and that the curvature should be adjustable during flight, has contributed to our present success at outperforming the avian teacher.}

A sense of perspective requires that some significant limitations of nature's lessons be described. Nature optimizes its materials for different functional requirements and operating environments than we do. Living organisms therefore are not regarded as a source of high-temperature materials. (It is also worth noting, however, that bacteria have been found under the conditions of high temperature and pressure-approximately $350^{\circ} \mathrm{C}$ and 265 atmospheres respectively-that prevail at hydrothermal vent sites on the ocean floor. It is an interesting prospect that such organisms might be harnessed to produce protein polymers that are at least as thermally stable as nylon.) Natural structural materials currently are known to consist of relatively few types of significantly different proteins, polysaccharides, and crystalline precipitates. Most of the naturally occurring elements are not present in these materials. The evolution of natural materials occurs as a long succession of small steps; materials evolve slowly as organisms tin- synthesis of nacre (or even to leave the impression that this is what they are doing); the rate at which a nacreous layer grows naturally is just too slow to be of any economic use to a materials engineer. On the other hand, it would also be highly questionable to copy only the very coarsest microstructural features of nacre in a synthetic ceramic/polymer composite, and then claim that the composite is in any sense superior to others because it has some features in common with a natural material. There is useful knowledge to be gained, however, by studying the molecular details of how the ceramic phase in naexpressing personal points of view on issues of interest to the materials community. cre (or teeth, bones, and other calcareous shell materials) is constrained to accumulate in a directed manner on organic templates. Thus, meaningful biomimicry identifies selected, worthwhile attributes to copy from a natural material. Another

\section{Useful practical lessons can and should be drawn from nature.}

illustration is afforded by the history of aviation. Many aspiring aircraft designers, including Leonardo da Vinci, attempted to learn the principles of sustained flight by watching birds. Some early flying machines were constructed to have flapping wings. Their failure constitutes an exercise in misdirected biomimetics. But learning that the curvature of a wing matters, and that the curvature should be adjustable during flight, has contributed to our present success at outperforming the avian teacher.

This discussion began by recognizing J.E. Gordon's pioneering, balanced appreciation for nature's lessons on optimized materials and structures. (Incidentally, he communicates this without recourse to buzzwords.) It might be fitting to conclude with another quote from Structures, that suggests how materials engineers could join aeronautical engineers in benefitting from ornithology: "I am inclined to think that the main advantage of feathers to an animal may be structural ... Feathers not only enable birds to get away with more local scrapes and abrasions than other animals, but the body of the bird is protected from more serious damage by its thick resilient armour. The Japanese feather armour which one sees in museums... was an effective protection against weapons like swords ... When one considers the appalling and expensive frequency with which motor cars nowadays incur bumps, bashes, and abrasions, one sometimes wonders whether they have not a lesson to learn from the birds." Clearly, there is a useful suggestion here. But the materials engineer who hurries to cover his automobile with feathers might be better off wearing them himself!

Christopher Viney is on the faculty of the University of Washington, Seattle. His primary appointment is in the Center for Bioengineering, and he holds an adjunct appointment in Materials Science and Engineering. 


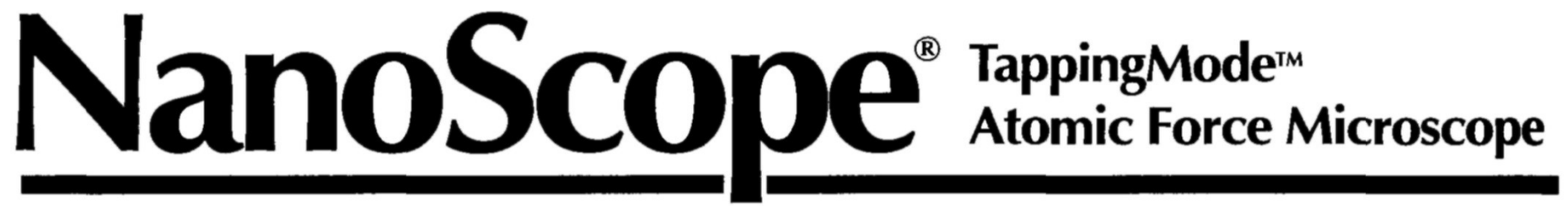

\section{Nondestructively Image and Measure Your Samples With 100-Times the Lateral Resolution of Stylus Profilers}

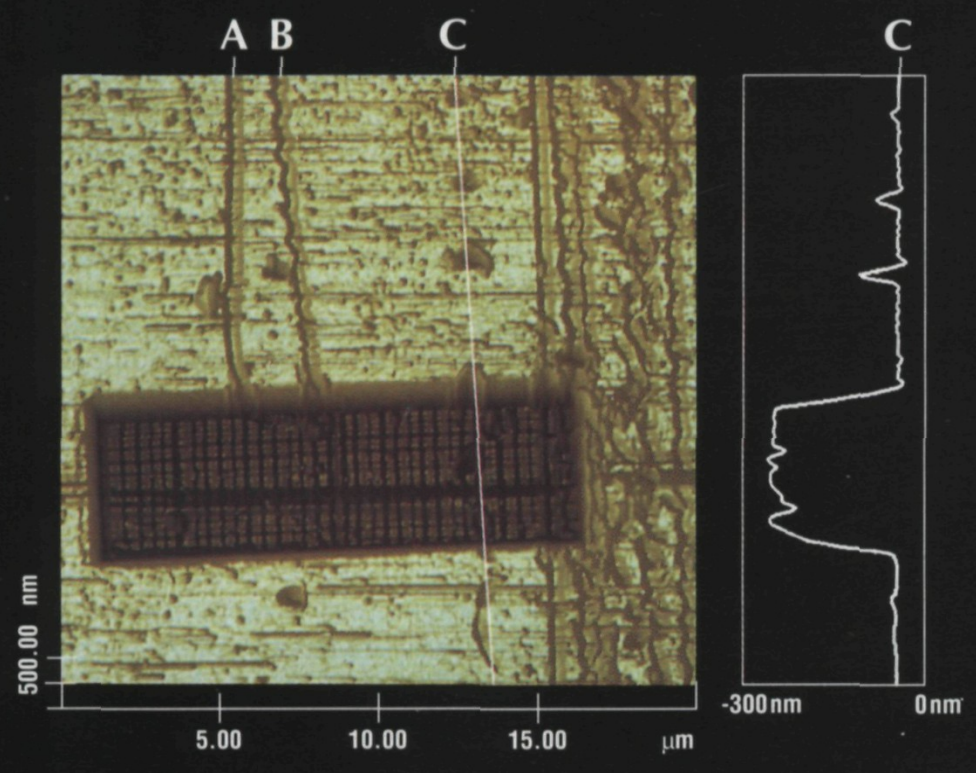

Measure Your Samples, don't Torture Them!

A NanoScope TappingMode ${ }^{T M}$ AFM Image and Cross Section of a Quartz Phase-shift Mask for Semiconductor Manufacturing. This scan shows damage to the sample left by a previous measurement with a popular surface profiler $(A, B, C)$. Notice how the stylus bounced as it came out of the trench, making a series of holes (C).

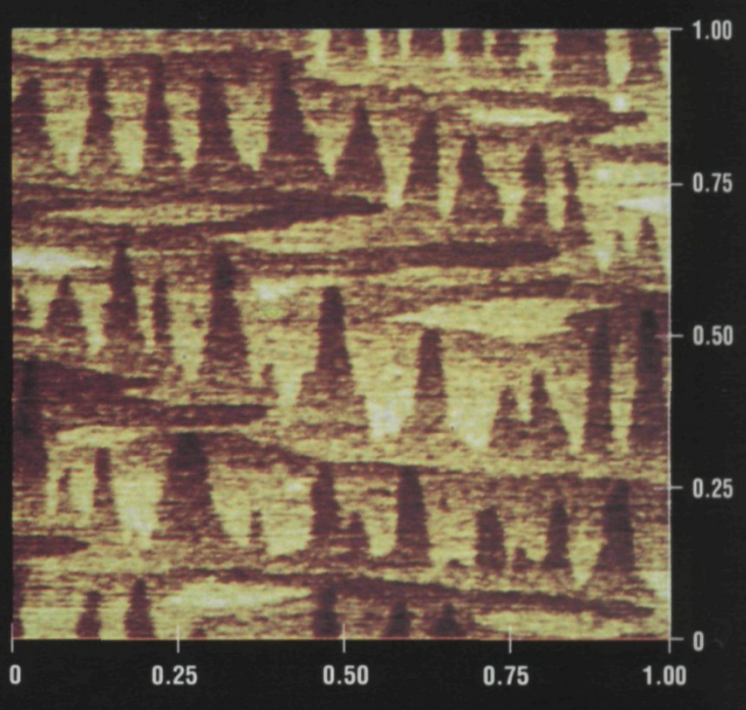

And See the Details You've been Missing!

A TappingMode ${ }^{\mathrm{TM}}$ AFM image of an intact 8 in Epitaxial Silicon Wafer. This scan shows 1.6 $\AA$ atomic steps - the expected value for the $\mathbf{1 0 0}$ plane of Silicon. A stylus profiler simply cannot see such fine details. Both these scans were made using the Nanoscope Large Sample Stage.
$\mathrm{D}$ igital Instruments, the world's leader in AFM Technology, brings you the capability to image and measure samples as no other technique can. Our exclusive TappingMode
AFM (patent pending) allows even soft and sticky samples to be imaged without damage. Call us today to discuss your application.

\section{Q 9 Digital Instruments}

Santa Barbara, CA •TEL: 805-899-3380 or 800-873-9750 -FAX: 805-899-3392 